Check for updates

Cite this: Phys. Chem. Chem. Phys., 2018, 20, 23085

Received 23rd May 2018, Accepted 19th July 2018 DOI: $10.1039 / c 8 c p 03281 \mathrm{e}$ rsc.li/pccp

\section{Mechanisms of reinforcement in polymer nanocomposites $\dagger$}

\author{
N. Molinari, (iD a A. P. Sutton ${ }^{a}$ and A. A. Mostofi (iD *ab
}

Coarse-grained molecular dynamics simulations are used to elucidate molecular mechanisms responsible for different mechanical behaviours of elastomers containing spherical particles with different volume fractions. We observe that different filler volume fractions result in qualitatively different responses of the polymer nanocomposite to tensile strain. At relatively low filler volume fraction a yield drop appears in the stress-strain curve. As the filler volume fraction increases there is a reduction in the rate of plastic hardening, becoming plastic softening at sufficiently high filler volume fraction. We demonstrate that these behaviours are a result of the network formed by the polymer chains and filler particles. We identify three distinct molecular structural motifs between polymer and filler particles whose relative prevalence varies with the filler volume fraction and as the system is dynamically strained. We show how this evolution in molecular structure is directly linked to the observed mechanical response.

\section{Introduction}

In effectively all practical applications, the mechanical, rheological and chemical properties of polymers are modified and enhanced by the inclusion of nanoparticle (NP) fillers. The wide range of shapes, dimensions and chemistries of NPs enables various properties to be tailored, including flammability, ${ }^{1,2}$ erosion resistance, $^{3,4}$ stiffness $^{5-7}$ and glass transition temperature. ${ }^{8-10} \mathrm{~A}$ deeper understanding of the molecular mechanisms underlying the improved properties induced by fillers is both of scientific interest and may enable the design and development of higher performance polymer formulations that better meet the requirements of their intended use. Computer simulations provide a framework to develop such an understanding, and in this paper we focus on the mechanical response of NP-filled polymer nanocomposites.

The mechanical properties of polymers without fillers have been studied with both all-atom ${ }^{11}$ and coarse-grained (CG) models. ${ }^{12-20}$ Whilst an all-atom approach is essential for understanding and predicting properties that depend on specific

\footnotetext{
${ }^{a}$ Department of Physics and the Thomas Young Centre for Theory and Simulation of Materials, Imperial College London, London SW7 2AZ, UK.

E-mail: nicola.molinari12@alumni.imperial.ac.uk,a.mostofi@imperial.ac.uk; Tel: +44 (0)20 75948154

${ }^{b}$ Department of Materials and the Thomas Young Centre for Theory and Simulation of Materials, Imperial College London, SW7 2AZ, UK

$\dagger$ Electronic supplementary information (ESI) available: Details on the equilibration procedure, filler volume fraction as a function of the number of inserted filler particles, distributions of new bonds per chain inserted by the cross-linking procedure at different filler loadings, reinforcement at low strain, and additional persistence maps. See DOI: 10.1039/c8cp03281e
}

chemical interactions, ${ }^{21}$ it is computationally impractical for realistic models of filled polymers because of the typical volume fractions, the sizes of nanoparticle fillers $(10 \mathrm{~nm}$ to $100 \mathrm{~nm}$ ), and the time-scales associated with the dynamics of such complex systems. As a result, there have been many computational studies of filled polymers using different levels of coarse-graining, as highlighted in recent reviews. ${ }^{22-25}$ Since its first use by Kremer and Grest to study the dynamics of entangled linear polymer melts, ${ }^{26}$ the bead-spring model has been widely adopted in both its basic formulation and with various extensions and generalisations. This model reproduces generic behaviour in good agreement with both theoretical predictions, such as the Rouse and reptation models, ${ }^{26,27}$ and experimental measurements while having a relatively simple formulation. Bead-spring models have been extensively used to study the mechanical properties of filled polymers. For example: Raos et $a l^{28,29}$ used a coarse-grained dissipative particle dynamics approach to study the viscoelastic properties of rubber filled with spherical particles at constant filler loading under oscillatory shear deformations; Shen et $a l^{30,31}$ investigated the straininduced non-linear mechanical behaviour of a polymer filled with spherical and grafted nanoparticles, showing that there exists an optimal grafting density and grafted chain length that helps to improve the dispersion of the grafted NPs; and Liu et $a .^{32}$ studied the effect of filler volume fraction (FVF) and polymer-filler interaction parameters on the mechanical response of reinforced elastomers and identified the existence of an optimal FVF for mechanical reinforcement in the presence of strong polymer-filler interactions.

Despite the aforementioned work, key aspects of the relation between polymer-filler network and mechanical reinforcement 
remain unanswered: what is the role played by the molecular structural motifs of the polymer-filler network on reinforcement? How does the polymer-filler network evolve during the straining, and with what consequences for the mechanical reinforcement? Is the appearance of a yield drop limited to very strong polymer-filler interactions, or can it stem from structural modifications of the polymer-filler network at high filler loadings?

In this paper, we use molecular dynamics simulations and a coarse-grained bead-spring model to elucidate the molecular mechanism of mechanical reinforcement in nanoparticle-filled polymer nanocomposites under uniaxial strain. These simulations are all performed above the glass transition temperature. We find that the stress-strain relation undergoes qualitative changes as a function of FVF, including the appearance of a yield drop. In ref. 32, this yield drop was attributed to strong filler-polymer interactions. In contrast, we observe that a yield drop begins to appear at relatively low FVF (31\%) and even when the polymer-filler interaction strength is less than half that of ref. 32. The central result of this work is that we find these and other variations in the stress-strain relations with FVF are all directly attributable to changes in the polymer-filler network, which we identify and quantify in both equilibrium configurations and dynamically during uniaxial straining. We identify three distinct local structural motifs, sketched in Fig. 1, the relative abundances of which vary systematically with FVF and strain. We show how this evolution in molecular structure is directly linked to the observed mechanical response.

\section{Model and simulation methods}

The coarse-grained model of the polymer used in this work is a development ${ }^{32}$ of the model proposed by Kremer and Grest ${ }^{26}$ to take account of filler particles. The total number of polymer chains in the system is set to 1000 , each comprising 30 beads of diameter $\sigma$ and mass $m$, unless otherwise specified. The bonds within a chain are represented by a combination of the finite
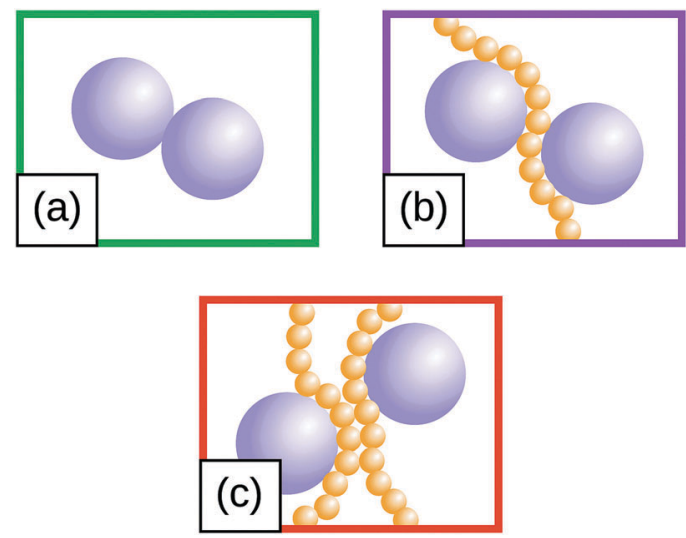

Fig. 1 (a)-(c) Sketch of the three predominant motifs observed at all filler loadings investigated. In (a) two filler particles are in direct contact, in (b) and (c) their interaction is mediated by one and two polymer chains, respectively. extensible nonlinear elastic (FENE) potential and a LennardJones (LJ) potential, as in previous studies. ${ }^{26,32}$ The analytic form of this bonded part of the potential is given by,

$$
\begin{aligned}
U^{\mathrm{B}}(r) & =U^{\mathrm{FENE}}(r)+U^{\mathrm{LJ}}(r) \\
& =-\frac{1}{2} k R_{\mathrm{B}}^{2} \ln \left[1-\left(\frac{r}{R_{\mathrm{B}}}\right)^{2}\right]+4 \varepsilon\left[\left(\frac{\sigma}{r}\right)^{12}-\left(\frac{\sigma}{r}\right)^{6}\right],
\end{aligned}
$$

where $r$ is the separation of two bonded beads and we choose $R_{\mathrm{B}}=1.5 \sigma$ and $k=30 \varepsilon / \sigma^{2}$ to avoid chain-crossing and high frequency modes. ${ }^{26,33}$ An average cross-link density of one cross-link per chain is used throughout this work and crosslink bonds have the same potential as in-chain bonds. Filler particles are represented by additional spheres with radius $R_{\mathrm{F}}=2 \sigma$. Polymer beads and filler particles are assumed to have the same mass density, therefore the mass of a filler particle is 64 times the mass of a polymer bead. Different filler volume fractions, or filler loadings, are simulated by generating systems which contain different numbers of filler particles in the structure, from unfilled to 1500 filler particles. For each filler loading, an ensemble of eight independently generated simulation boxes are created (as described later) and all computed quantities are averaged over the ensemble. As elsewhere in the literature, ${ }^{34,35}$ the bead-bead, bead-filler and filler-filler non-bonded interactions are modelled with a truncated and shifted LennardJones potential (TSLJ):

$$
\begin{aligned}
& U_{\mathrm{TSLJ}}^{i}(r) \\
& = \begin{cases}4 \alpha_{i}\left[\left(\frac{\sigma}{r-\Delta_{i}}\right)^{12}-\left(\frac{\sigma}{r-\Delta_{i}}\right)^{6}\right]-U_{\mathrm{LJ}}\left(r_{i}^{\mathrm{c}}\right) & \Delta_{i}<r \leq r_{i}^{\mathrm{c}}+\Delta_{i} \\
0 & r>r_{i}^{\mathrm{c}}+\Delta_{i} \\
+\infty & 0<r \leq \Delta_{i}\end{cases}
\end{aligned}
$$

where $i$ represents the type of non-bonded interaction (beadbead, bead-filler or filler-filler), each with its own set of values for $\alpha_{i}, \Delta_{i}$ and $r_{i}^{\mathrm{c}}$, as summarised in Table $1 . \alpha_{i}$ controls the strength of the interaction, $\Delta_{i}$ shifts the interaction to take into account the excluded volume effects, and $r_{i}^{\mathrm{c}}$ is the distance at which the interaction is truncated and shifted so that the force and energy are zero. As filler particles are often made of silica, clay, and carbon black, they are assumed to exhibit negligible attraction to each other. Therefore, as in previous studies, ${ }^{32}$ fillers interact with other fillers as hard spheres, making their TSLJ potential equivalent to a repulsive WCA potential. ${ }^{36}$

Table 1 Summary of potential parameters used in this work for the nonbonded interaction potential, where $\alpha_{i}, \Delta_{i}$ and $r_{i}^{\mathrm{c}}$ are defined in the main text. $R_{\mathrm{F}}$ is the radius of the filler particles, and it is kept fixed at $R_{\mathrm{F}}=2 \sigma$ throughout this work

\begin{tabular}{llll}
\hline$i$ & $\alpha_{i} / \varepsilon$ & $\Delta_{i}$ & $r_{i}^{\mathrm{c}} / \sigma$ \\
\hline Bead-bead & 1.0 & 0 & $2 \sqrt[6]{2}$ \\
Bead-filler & 5.0 & $R_{\mathrm{F}}-\sigma / 2$ & $2 \sqrt[6]{2}$ \\
Filler-filler & 1.0 & $2 R_{\mathrm{F}}-\sigma$ & $\sqrt[6]{2}$
\end{tabular}


Previous studies, e.g., ref. 32, have investigated the effect of varying the bead-filler interaction and have shown that an interaction strength of at least twice the bead-bead interaction is necessary for the polymer chains to wet the filler surface and provide mechanical reinforcement. We chose the (attractive) bead-filler interaction strength to be five times the bead-bead interaction strength so that the system is clearly within the region in which fillers result in mechanical reinforcement, and with the aim of modelling relatively strong polymer-filler interfacial interactions that would result from, for instance, functionalisation of the filler particles. The shift used to account for the excluded volume of the fillers is chosen such that $\Delta_{i}=R_{1}+R_{2}-\sigma$, where $R_{1}$ and $R_{2}$ are the radii of the two particles taking part in the interaction. With this choice, the zero of the TSLJ is shifted at a distance equal to that of the sum of the two radii.

We work with LJ units throughout, in which the mass $m$, and LJ parameters $\sigma$ and $\varepsilon$ are set to unity. All molecular dynamics simulations are performed using the Large-scale Atomic/ Molecular Massively Parallel Simulator (LAMMPS) package. ${ }^{37}$ A velocity-Verlet integrator with a timestep of $\delta t=10^{-3}$ was used to evolve the equations of motion. The reduced temperature was set to $T=1.0$ (well above the glass transition temperature for the system ${ }^{38}$ ) and the pressure to $P=0.0$ using a NoséHoover thermostat and barostat, respectively. The choice of temperature allows the system to undergo long-range co-ordinated motions and relaxations typical of a real elastomer above its glass transition, while the pressure is set to mimic the low ambient pressure.

The structures used in this investigation are created as in an earlier work, ${ }^{39}$ here we present a brief summary. First, the filler particles are randomly positioned one at a time in a large periodic box. If a filler particle overlaps with any of the previously placed ones, a new trial position is generated and tested. The dimension of the box is chosen so that, once all the components are inserted, the resulting density is equal to roughly $30 \%$ of the equilibrium density for the unfilled system, which is $\rho \approx 0.85 .^{26}$ Then the chains are added one bead at a time in a self-avoiding fashion in the remaining available space. Once the first bead of a new chain is successfully placed, the next suggested bead placement is done randomly on a sphere of radius $0.96 \sigma$ (minimum of $U^{\mathrm{B}}$, eqn (2)) centred on the previous bead. If the trial location does not overlap with any previously placed element, it is accepted and the neighbour lists are updated. The process is repeated until the desired number of beads per chain is reached. The whole procedure is then repeated for 1000 chains. The large initial volume of the simulation cell ensures that the process of generating the initial configuration of fillers and beads is computationally efficient and does not stagnate as a result of packing constraints. The structures then undergo an equilibration procedure comprising energy minimisation, compression, decompression, and annealing stages that is based on approaches adopted in previous studies. ${ }^{39-41}$ A detailed description is provided in the ESI, $\dagger$ Section 1. The minimisation and compression stages of the equilibration procedure are expected to take the structures from low to melt density.
Once these initial structures are equilibrated, cross-links are introduced between polymer chains. Bonds are added dynamically during a simulation with a constant number of particles, pressure and temperature (NPT) that automatically stops when the total number of added bonds is equal to 1000, i.e., to the set target of one cross-link per chain on average. Pairs of monomers within a distance of $1.25 \sigma$ are flagged as potential crosslink sites if they do not already share a bond. If the condition is satisfied, the probability to create a cross-link bond is set to $0.03 \%$. This choice of the probability of cross-linking avoids inserting too many new bonds at the same timestep, but it also prevents stagnation of the cross-linking procedure. Due to the stochastic nature of the cross-linking of the structures, it is important to verify that no bias is introduced among structures with different numbers of inserted nanoparticles. To this end, we computed the distribution of added bonds (i.e. cross-linking bonds) per chain for all eight structures at each filler loading. We observed no significant difference among the distributions of new bonds at different filler loadings. The full results are shown in the ESI, $\dagger$ Section 3. To relax the new bonds, and any structural modifications introduced, the systems are re-equilibrated following the same procedure as before. The FVF reported for each system is calculated at this stage with a 100000 timesteps NPT simulation $(T=1.0$ and $P=0.0)$, where the density is recorded every 100 timesteps. Within the ensemble of structures sharing the same number of inserted filler particles, the variations in the average FVFs are very small compared to the standard deviations, and are therefore neglected. As a result, the eight structures with the same number of fillers are treated as having the same, mean FVF and used to compute ensemble averages at constant FVF throughout this work. A plot of the FVF as a function of the number of inserted filler particles can be found in the ESI, $\uparrow$ Section 2.

The polymer-filler systems, prepared as described above, are uniaxially strained along the $z$-axis within the $N T L_{z} \sigma_{x x} \sigma_{y y}$ ensemble, in which the total number $N$ of particles and temperature $T$ are kept constant, and normal stresses $\left(\sigma_{x x}\right.$ and $\left.\sigma_{y y}\right)$ perpendicular to the straining direction are kept zero. ${ }^{42}$ During the straining procedure $L_{z}$, the length of the simulation box along the straining axis, is increased at a uniform strain rate $\dot{\eta}$, such that $L_{z}(t)=L_{z}(0)(1+\dot{\eta} t)$, whilst the lateral stresses $\sigma_{x x}$ and $\sigma_{y y}$ are kept zero. This mimics the usual boundary conditions on an experimental tensile test at a constant strain rate. As in previous studies with similar models $^{32,39,43,44}$ we apply a strain increment of $3.27 \%$ every $\tau=\frac{1}{\delta t}=10^{3}$ timesteps; i.e. the simulation box is strained along $z$ by $0.00327 \%$ every timestep $\delta t$. The straining is performed for a time of $100 \tau$, resulting in a final strain on the system of $327 \%$. The stress along the straining direction, $\sigma_{z z}$, is given by the negative of the pressure along the $z$ axis, $P_{z z}$. At each FVF, the reported stress at a given strain is averaged over eight independently generated systems. The width of the shading of the stress-strain curves below signifies $\pm 1 \sigma_{\mathrm{S}}$, where $\sigma_{\mathrm{S}}$ is the standard deviation of the calculated stresses. 


\section{Results and discussion}

\section{Stress-strain response as a function of filler loading}

Stress-strain curves corresponding to $0.0 \%, 27 \%$ and $47 \%$ FVFs are shown in Fig. 2(a), (b), (c), respectively, and Fig. 2(d) and (e) show examples of structures at the beginning and end of the straining procedure for $0.0 \%$ and $47 \%$ FVF, respectively. Fig. 2(a) serves as a reference point for the mechanical effects of fillers embedded in the polymer matrix since this corresponds to the unfilled case. In the stress-strain relation at $27 \%$ FVF, Fig. 2(b), quasi-elastic (up to 5\% strain) and plastic (for strains greater than $5 \%$ ) regions are clearly distinguished. At $47 \%$ FVF, Fig. 2(c), there is a yield drop following the initial quasi-elastic rise, as indicated by the appearance of a peak in the stress. A yield drop has been observed in earlier work, but only in the limit of very strong polymer-filler interactions (corresponding to a value of $\alpha_{\text {Bead-filler }}=12.0$ ). ${ }^{32}$ At strains between $100 \%$ and $250 \%$, the stress is almost independent of strain, in contrast to the case of $27 \%$ FVF where the stress increases with strain throughout the plastic region. At strains exceeding $250 \%$ there is the onset of necking eventually leading to fracture, as shown in Fig. 2(e). The standard deviation in the mechanical response among the eight structures at all FVFs is generally small, indicating a weak dependence of the stressstrain curve on the initial structures with the same FVF. This observation, which holds true throughout this study, gives us confidence that the number of independently-generated structures at every filler volume fraction is sufficiently representative, and the results statistically robust.

For FVF up to $27 \%$, the filled polymer is progressively strengthened by the inclusion of more particles, Fig. 3. The quasi-elastic region exhibits both a progressively higher modulus and higher peak before the onset of the plastic region (see the ESI, $\dagger$ Section 4,

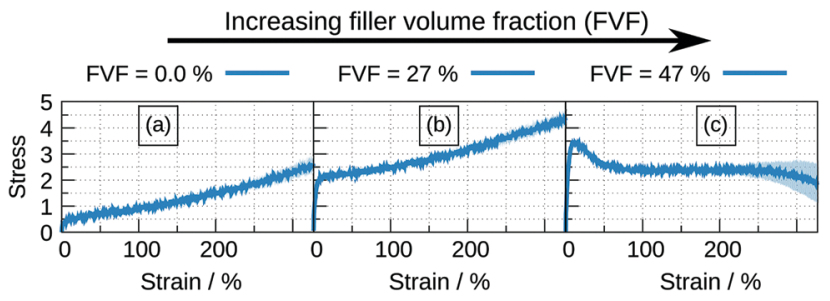

$0.0 \%$ Filler volume fraction $47 \%$ Filler volume fraction

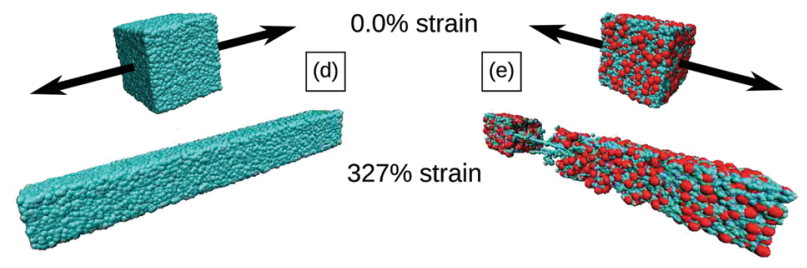

Fig. 2 Mechanical responses of elastomer nanocomposites with different FVF. $(a-c)$ show stress-strain curves for three FVFs exhibiting different responses to the loading. (d) and (e) are examples of structures at the beginning and end of the straining procedure. (d) is a structure with no filler loading while (e) has a 47\% FVF. In the latter necking can be observed. Note red spheres are filler particles, while the blue spheres represent the polymer beads.

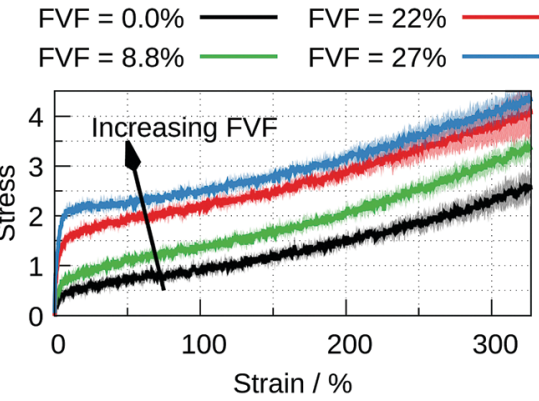

Fig. 3 Stress-strain curves for four FVFs, corresponding to $0.0 \%$ (black), $8.8 \%$ (green), $22 \%$ (red), and $27 \%$ (blue).

for an enlarged stress-strain plot up to $25 \%$ strain). There is no substantial change in the range of strain over which the stress-strain curve is quasi-elastic, and there is no substantial change in the slope of the plastic region. At larger FVF the stress-strain relation is merely displaced to higher stresses for the same strain. A different picture emerges with FVFs larger than $27 \%$. In Fig. 4, the stress-strain curves for $27 \%, 31 \%$, and $35 \%$ FVF are presented. At 31\% FVF a yield drop appears, which is a new feature in the stress-strain relation. This yielding phenomenon appears in all the stress-strain relations with FVF larger than $31 \%$, and becomes increasingly prominent with increasing FVF. Recalling that the strength of the polymer-filler interaction is the same at all FVFs, the presence of a threshold in the FVF before a yield drop appears suggests it is related to a change in the molecular structure of the nanocomposite at a critical FVF.

The smaller slope of the plastic region for $35 \% \mathrm{FVF}$, seen in Fig. 4, suggests the existence of an optimal FVF for mechanical reinforcement, where the peak of the quasi-elastic region is maximized before a reduction in the rate of hardening in the plastic region occurs. In this work the optimum is somewhere between $31 \%$ and $35 \%$ FVF. There are similar results reported elsewhere in the literature. ${ }^{32}$ To understand the origin of the variation of mechanical properties as a function of filler loading we investigate the molecular environments of filler particles in the next section.

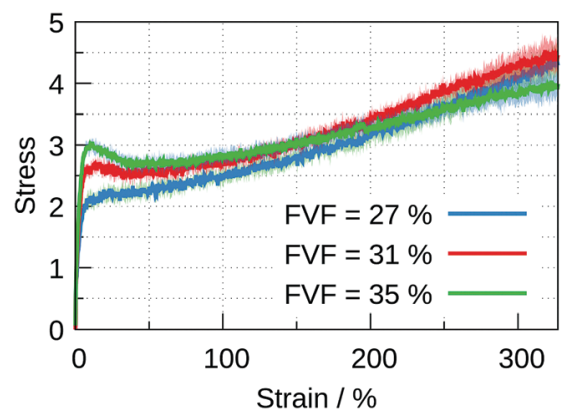

Fig. 4 Stress-strain curves for three FVFs of 27\%, 31\%, and $35 \%$. Up to $27 \%$ FVF (blue) the mechanical response does not exhibit a yield drop. At $31 \%$ FVF (red) a peak appears between the quasi-elastic and plastic regions. At $35 \%$ FVF (green) the peak is clearly present and the slope of the plastic region becomes smaller compared with that of lower FVFs. 


\section{Polymer-filler network at equilibrium (0\% strain)}

The radial distribution functions (RDF) between filler particles are calculated in all structures at all FVFs. The RDFs are computed after the structures are equilibrated, but before being strained. For each equilibrated structure, a total of 50 snapshots separated by $100 \delta t=0.1 \tau$ are recorded and used to compute the average RDF of a single structure. Fig. 5 shows examples of the RDF for one representative structure for the cases of $27 \%$ and $40 \%$ FVF. The distributions show peaks corresponding to three molecular structural motifs: (1) fillers in direct contact with each other, Fig. 1(a), with corresponding peak at $2 R_{\mathrm{F}}=4 \sigma$; (2) fillers separated by one layer of polymer beads, Fig. 1(b), with corresponding peak at $2 R_{\mathrm{F}}+\sigma=5 \sigma$; and (3) fillers separated by two layers of beads Fig. 1(c), with corresponding peak at $2 R_{\mathrm{F}}+2 \sigma=6 \sigma$. These findings are in good agreement with the classification of the dispersion state of nanoparticles by Hooper and Schweizer. ${ }^{45}$ In particular, the structural motifs found in Fig. 1(a)-(c) can be interpreted as contacts of type: (i) direct contact, (iii) segmental level tight particle bridging, and (iv) "tele-bridging", respectively. The area under each peak is a measure of the prevalence of the corresponding molecular structural motif (or MSM), and it is calculated as in eqn (4):

$$
A=\frac{4 \pi N_{\mathrm{F}}}{V} \int_{r_{1}}^{r_{2}} r^{2} g(r) \mathrm{d} r,
$$

where $r_{1}$ and $r_{2}$ are two consecutive minima of $g(r)$, or RDF, $V$ is the box volume, and $N_{F}$ the number of filler particles. In Fig. 6 , we show this quantity as a function of the FVF, where the value reported for each FVF is an average over an ensemble of eight independently generated structures. The label BB, which stands

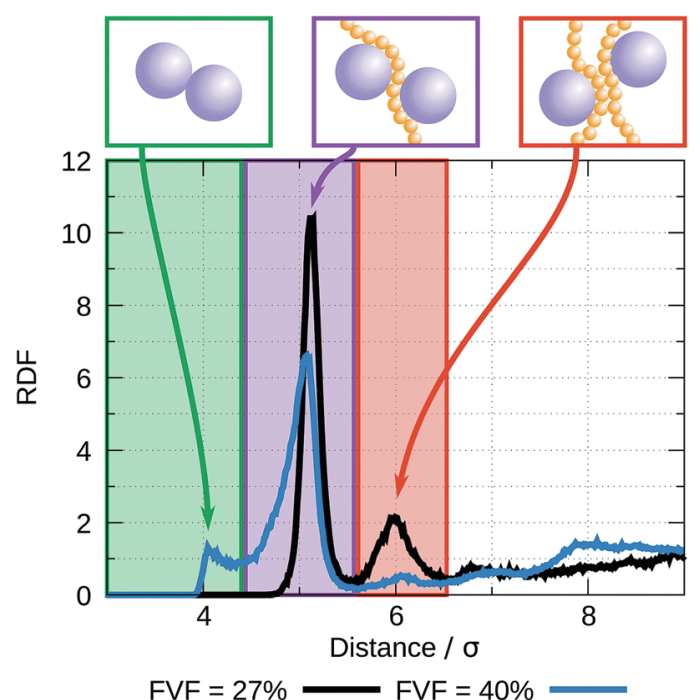

Fig. 5 Examples of RDFs: black corresponds to a 27\% FVF structure, and blue to a $40 \%$ FVF structure. The former shows peaks at $5 \sigma$ and $6 \sigma$ that correspond to fillers separated by one and two layers of polymer beads, respectively. The RDF for the $40 \%$ FVF structure shows a peak at $4 \sigma$, which relates to fillers in direct contact with each other, a peak at $5 \sigma$ and a very small bump at $6 \sigma$.

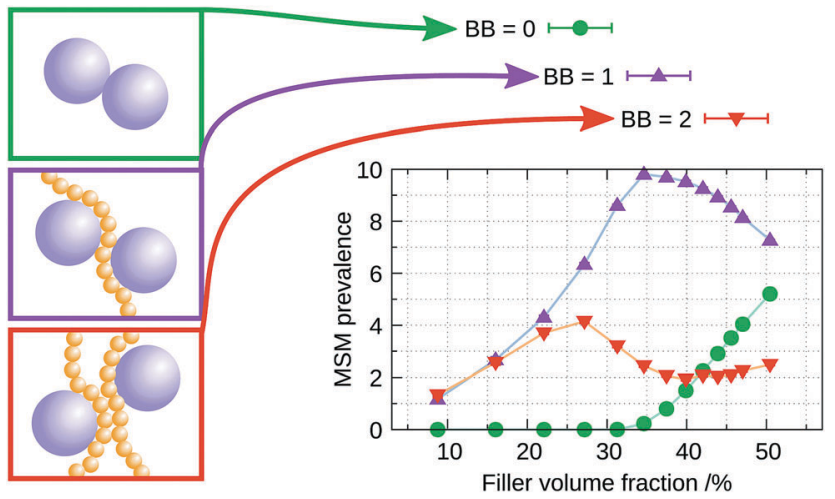

Fig. 6 Prevalence of the different peaks in the filler-filler RDF as a function of FVF. The three curves correspond to the first three peaks in the distribution that indicate: (green) fillers touching directly $(B B=0)$, (purple) fillers with one intervening layer of polymer beads $(B B=1)$ and (red) fillers with two intervening layers of polymer beads $(B B=2)$. The error bars are the standard deviations among eight independently generated structures.

for "bridging beads", denotes the number of polymer bead layers between two fillers and is used to distinguish the different molecular structural motifs henceforth. Three main regions are identified:

- Up to $27 \%$ FVF: the filler-filler interactions are almost entirely mediated by either one $(\mathrm{BB}=1)$ or two $(\mathrm{BB}=2)$ bridging polymer bead layers. As the FVF increases up to $27 \%$, the prevalence of both $\mathrm{BB}=1$ and $\mathrm{BB}=2$ increase. A negligible fraction of filler particles are in direct contact $(\mathrm{BB}=0)$ and there is no discernible peak corresponding to $\mathrm{BB} \geq 3$. Polymer beads are attracted to filler particles because the strength of their interaction is five times that of the bead-bead interaction, see Table 1.

- Between 27\% and 35\% FVF: BB = 2 peak falls rapidly. The relative abundance of $\mathrm{BB}=1$ continues to increase through this range of FVFs, reaching a maximum at $35 \% \mathrm{FVF}$, while $\mathrm{BB}=0$ starts to appear at $35 \% \mathrm{FVF}$.

- Above 35\% FVF: $\mathrm{BB}=0$ rises and $\mathrm{BB}=1$ falls, and they almost meet at $50 \% \mathrm{FVF} . \mathrm{BB}=2$ remains approximately constant and small throughout this range.

As shown above, the network formed by the polymer chains and the filler particles at equilibrium ( $0 \%$ strain) varies as a function of FVF. This variation correlates qualitatively with the mechanical response for different FVFs, as determined by the stress-strain curves (Fig. 2-4). As the $\mathrm{BB}=2$ molecular structural motif falls rapidly, a peak in the stress-strain response appears. $\mathrm{BB}=1$ reaches a maximum at approximately $35 \% \mathrm{FVF}$, while $\mathrm{BB}=0$ remains absent but starts to appear at $35 \% \mathrm{FVF}$, which coincides with the observed weakening of the plastic regime. This observation indicates the mechanical response at large strains is optimised at this filler loading. In other words, as the slope of the plastic region is decreased beyond 35\% FVF, for optimal reinforcement at high strains the filler loading should be lower than $35 \%$. These observations indicate that the static picture provided by a snapshot of the organisation of the filler-polymer network at equilibrium 
( $0 \%$ strain) determines to a large extent the nature of the dynamic stress-strain relation. However, for a more detailed understanding of the molecular mechanisms of mechanical deformation, it is necessary to study explicitly the dynamical evolution of the polymer-filler network as it undergoes strain.

\section{Coordination changes during straining}

The calculation of the RDF is performed similarly to the static case, but adapted to be computed dynamically during the straining of the structures. The only difference with the static case is that the averaging at a given strain is performed on a single snapshot in eight dynamically evolving structures at that strain, rather than over 50 snapshots. This is done to capture the instantaneous distribution of polymer chains and filler particles. As in the static case, the integrals of the peaks of the RDF are averaged over eight structures from independent runs of the straining process. The larger number of beads in our model, as compared with previous work in the literature, ${ }^{32}$ results in a higher number of filler particles at a given FVF. This translates into well-converged RDFs even without averaging over multiple snapshots. $\neq$ As a result, the instantaneous RDF, and hence the filler-polymer network structure, can be captured dynamically during the straining process. Fig. 7 shows the distribution of the molecular structural motifs in the system up to $100 \%$ strain, and for different FVFs. It is seen that at strains up to about $\sim 5 \%$ the prevalences of the molecular structural motifs remain approximately, but not exactly, constant. This is why we call the initial increase of stress from zero quasi-elastic: there are a few coordination changes happening, which are presumably irreversible. The principal features in the mechanical response, i.e., the appearance of a yield drop and the weakening of the plastic region observed in Fig. 4, are now discussed in the light of the dynamical evolution of the coordination motifs.

Yield drop. As previously observed in Fig. 4, a yield drop is absent in structures with FVF $\leq 27 \%$, but it appears in all the stress-strain relations for structures with FVF $>31 \%$. The condition for a yield drop to arise is that there is a mechanism to enable stretching of the polymer-filler network at lower stresses once the plastic region has been entered. In Fig. 7 at 16\% FVF all three molecular structural motifs change little. But at $31 \% \mathrm{FVF}, \mathrm{BB}=1$ decreases with strain as seen in Fig. 7(c). For BB $=1$ a polymer chain is sandwiched between two filler particles and it experiences enhanced attraction to both fillers. This restricts the movement of the polymer. Upon straining, stress builds up until the polymer molecule detaches from one of the filler particles, remaining attached to the second. The polymer molecule is then less constrained and further deformation can take place at lower stresses. At the same time in nanocomposites with FVFs $\geq 31 \%$ the MSMs BB = 0,2 tend to increase, which further reduces the constraints on the polymer network. To summarise, it is the decline of the constrained $\mathrm{BB}=1$ population and the growth of the $\mathrm{BB}=0$ and/or 2 populations during straining that results in the yield drop.

Plastic weakening. For FVFs greater than $31 \%$, the strength of the nanocomposite tends to decline with increasing strain in

\# With the only exception being the lowest FVF $(8.8 \%, 100$ filler particles). the plastic region as observed in Fig. 4. The depletion of the $\mathrm{BB}=1 \mathrm{MSM}$ during the straining process results in an increased number of fillers in direct contact, as seen by the increase in the $\mathrm{BB}=0 \mathrm{MSM}$ in Fig. $7(\mathrm{~d})$. As the volume fraction of the energetically weakest $\mathrm{MSM}, \mathrm{BB}=0$, increases with strain so the slope of the stress-strain curve declines. This explains the observed decline in the strength of the nanocomposites with increasing strain in the plastic regions for FVFs exceeding 31\%.

\section{Average and total filler coordination}

Further insight into the molecular mechanisms behind the evolution of the stress-strain behaviour as a function of FVF can be obtained by considering the average and total coordination of filler particles by polymer beads. The evolution during straining of the average and standard deviation of the number of polymer beads coordinating a filler particle are plotted in Fig. 8 for a range of FVFs. At $8.8 \%$ and 16\% FVFs the number of beads coordinating a filler particle stays approximately constant at 75. At higher FVFs the average number of beads coordinating a filler particle initially declines quite rapidly, but the rate of decrease diminishes with further strain, until it is almost constant, amounting in each FVF to a loss of approximately eight coordinating beads by $100 \%$ strain. A key change occurs between $31 \%$ and $35 \%$ FVF: the average coordination is lower in $35 \%$ FVF at zero strain and remains smaller than in $31 \% \mathrm{FVF}$ at all subsequent strains. A lower average coordination signifies a weakened network, consistent with the declining strength of the nanocomposites with increasing strain in the plastic region observed for structures with FVF greater than $35 \%$. This observation is also consistent with the increasing prevalence of $\mathrm{BB}=0$ for FVFs greater than $31 \%$ observed in Fig. 6. A higher number of filler particles in direct contact results in a smaller fraction of the total filler surface area available to interact with polymer beads.

To elucidate why the highest stress attained in the quasielastic region continues to increase with increasing FVF, the stress at $10 \%$ strain is shown in Fig. 9 as a function of the total number of filler-polymer interactions. We see that despite the abundance of the $\mathrm{BB}=0$ molecular structural motif in the range $35 \%$ to $47 \% \mathrm{FVF}$, and the concomitant lower average coordination per filler particle observed in Fig. 8, the total number of polymer beads coordinating with fillers continues to increase with increasing FVF because there are more filler particles, resulting in an increasing stress at $10 \%$ strain with increasing FVF. It is also apparent that for FVFs up to $35 \%$ the maximum stress increases linearly with the total number of coordinating beads. The deviation from the linear relationship observed for $47 \%$ FVF can be correlated with the relatively high fraction of the $\mathrm{BB}=0 \mathrm{MSM}$ present at this FVF, which weakens the network.

\section{Persistence and average coordination}

Whilst answering important questions, the average and total general coordination of the fillers by the polymer beads does not elucidate the evolution of the specific polymer beads coordinating the filler particles. In this section we investigate the dynamical nature of changes in the coordination of a filler 


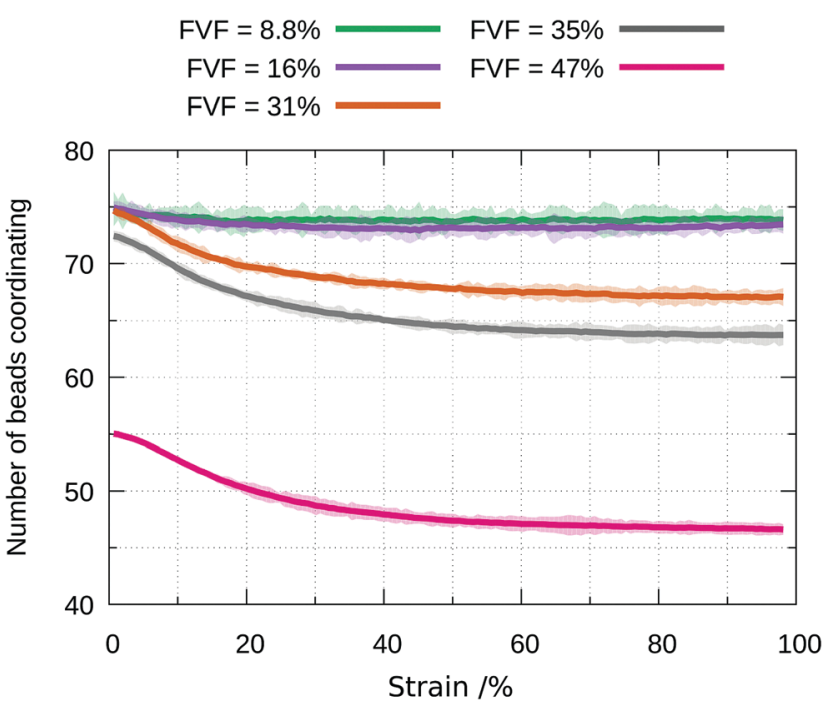

Fig. 8 Evolution during straining of the average total number of beads coordinating filler particles in a range of FVFs. The shading indicates the standard deviation.
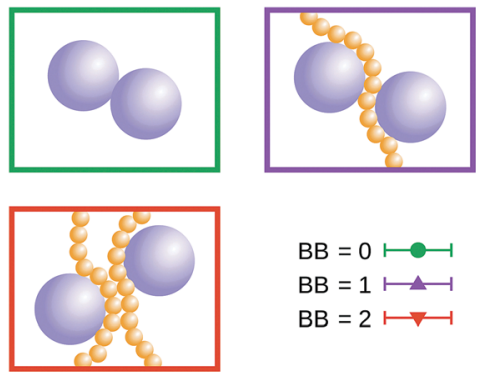

$\mathrm{BB}=0 \longmapsto$

$\mathrm{BB}=1 \longmapsto$

$\mathrm{BB}=2 \longmapsto$
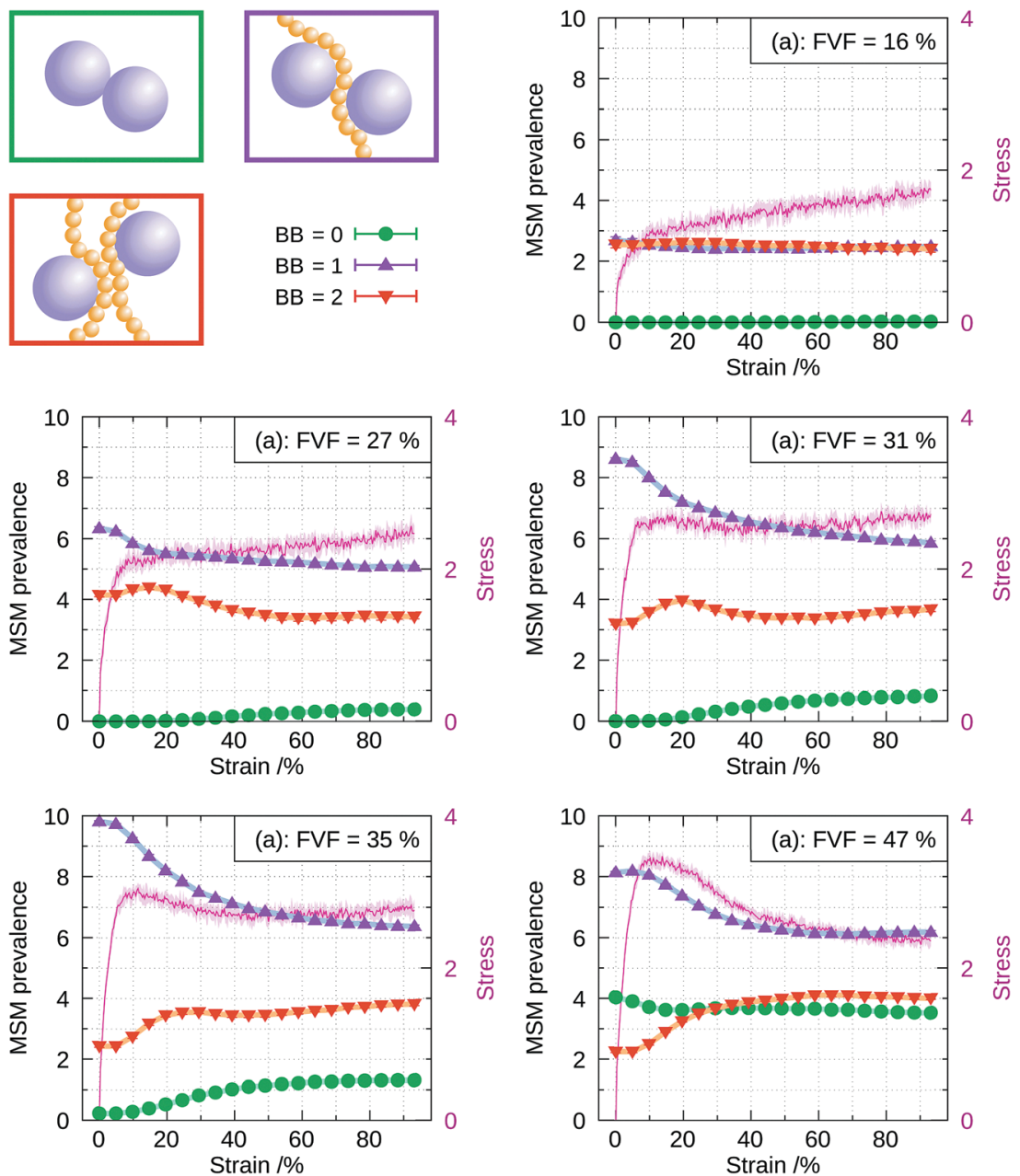

Fig. 7 Prevalence of the molecular structural motifs obtained by integration of the filler-filler RDF at different FVFs and straining. The error bars are the standard deviations among eight independently generated structures. The stress-strain relations are also plotted (magenta) for ease of reference.

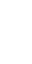

standard deviations among eight independently generated structures. The stress-strain relations are also plotted (magenta) for ease of reference. 


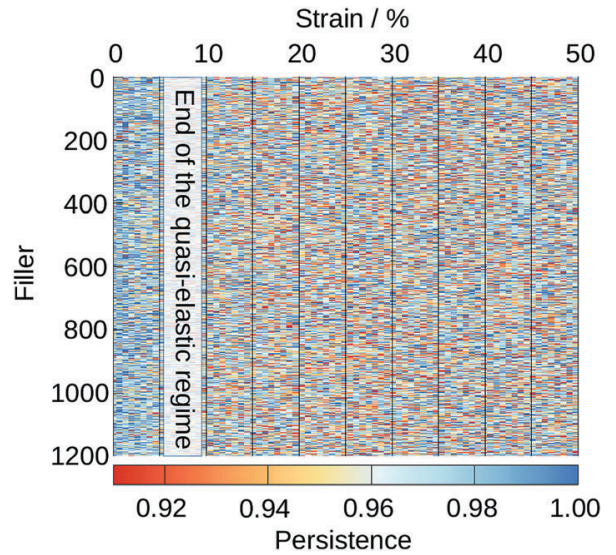

Fig. 10 Persistence of each of the 1200 fillers in a 47\% FVF structure as a function of strain. Similar plots for the other seven $47 \%$ FVF structures, are presented in the ESI $\dagger$ Section 5, where it is seen they display the same general features as seen in this plot.

different sets of polymer beads are coordinating the filler particles in the two snapshots. Since the two snapshots are $0.1 \tau$ apart, we expect the persistence to be unity or only slightly less than unity. If a bead is within $R_{\mathrm{F}}+\sigma=3 \sigma$ from the centre of a filler, it is defined as coordinating. The distance of $3 \sigma$ is chosen because it corresponds to the first minimum of the filler-bead RDF. Let $G_{n}^{i}$ and $G_{n+1}^{i}$ be the sets containing the indices of the beads coordinating filler $i$ at straining steps $n$ and $n+1$, respectively. Then the persistence for filler $i$ at straining step $n+1, \mathcal{P}_{n+1}^{i}$, is defined as follows:

$$
\mathcal{P}_{n+1}^{i}=\frac{G_{n}^{i} \cap G_{n+1}^{i}}{\left|G_{n}^{i}\right|} .
$$

Fig. 10 shows the evolution of the persistence as a function of strain for each of the 1200 fillers in a representative structure with $47 \%$ FVF. The corresponding plots for the other seven $47 \%$ FVF structures can be found in the ESI, $\dagger$ Section 5 .

There are two important features observed in Fig. 10. First, although rare, there are regions with a persistence $\sim 91 \%$ at strains less than $5 \%$. This confirms our earlier statements that in the quasi-elastic region there are fillers that undergo changes in their coordinations with polymer beads, which imply this region cannot be truly elastic, i.e., reversible. At strains greater than $5 \%$ changes in the coordination of filler particles are more frequent and are experienced by all filler particles.

Second, close inspection of Fig. 10 reveals that changes in the local coordination environments of a given filler particle do not last longer than a few straining steps. Once the local coordination of a filler is reduced, stress is transferred to other fillers that may then undergo similar transitions, while the coordination environment of the first filler is temporarily constant. And so the cycle of disrupting and conserving the coordination environment of each filler particle continues.

In Fig. 11 we show for each FVF the value of the persistence averaged over all filler particles as a function of strain. The average persistence for $8.8 \%$ and $16 \%$ FVF stays almost

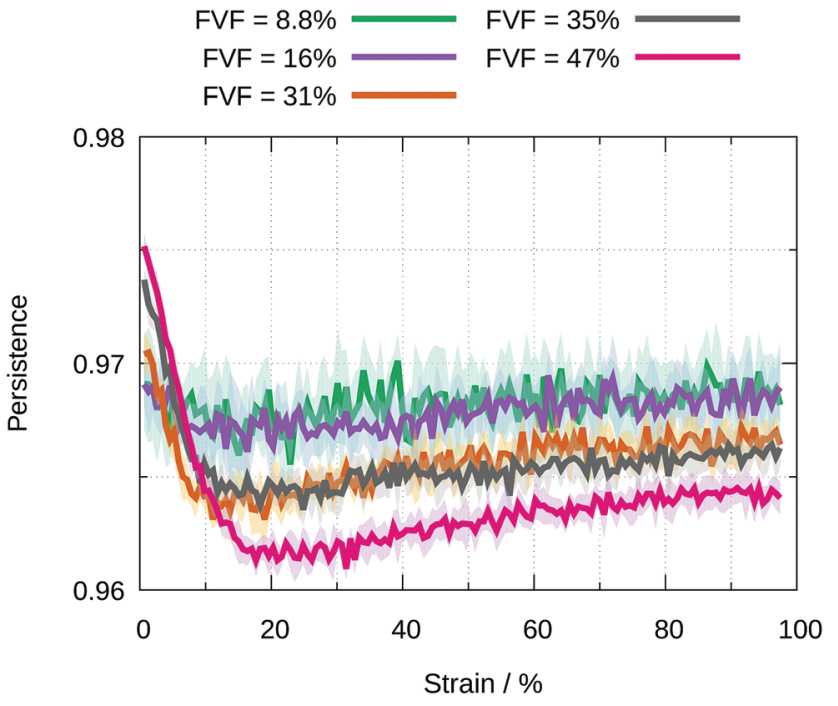

Fig. 11 Average value of the persistence for all filler particles at a given FVF as a function of strain. The standard deviations are shown by the shading.

constant at around 97\%, indicating small changes in the specific beads coordinating the fillers. The persistence at strains less than 5\% increases with increasing FVF because the network is stabilised by the increasing number of the $\mathrm{BB}=1$ molecular structural motif, as seen in Fig. 6. For FVF of $31 \%$ and above, the average persistence decreases sharply in the strain range $0 \%$ to $20 \%$ and recovers somewhat at larger strains. It is interesting that the disruption of the network at strains less than $20 \%$ and its increasing stabilisation at larger strains coincides with the appearance of the yield drop.

\section{Conclusions}

In this work we have used coarse-grained molecular dynamics to simulate tensile tests of polymer nanocomposites containing spherical filler particles for a range of filler volume fractions (FVFs) from $0 \%$ to $\sim 50 \%$. We have assumed the interaction between polymer beads and filler particles is five times stronger than that between polymer beads to model nanocomposites where the surfaces of filler particles have been modified to enhance adhesion to the polymer.

At all finite FVFs we find the stress-strain relation comprises an initial quasi-elastic region, characterised by a steep slope, followed by a plastic region where the slope is significantly smaller. There is not a sharp transition between these two regions but in all cases the extent of the quasi-elastic region is no more than the first $10 \%$ of strain. As seen in Fig. 7 the maximum stress reached in the quasi-elastic region increases with increasing FVF. This is a result of the monotonic increase of the total number of interactions between polymer beads and filler particles with increasing FVF, shown in Fig. 9. The term quasi-elastic is used because there are changes in the coordination environments of fillers at strains up to $10 \%$ (Fig. 10). These changes are infrequent compared to those taking place in the 
plastic region, but their existence indicates a small but finite degree of irreversibility and hence plasticity.

In the plastic region there is no yield drop for FVFs up to $27 \%$. The slope of the stress-strain relation remains positive (Fig. 3). At $31 \%$ FVF a yield drop appears which becomes more pronounced with increasing FVF. At 35\% FVF the slope of the stress-strain curve in the plastic region begins to decrease with increasing FVF, becoming negative at all strains in the plastic region at $47 \% \mathrm{FVF}$ (Fig. 7). We attribute both the yield drop and the weakening of the plastic region to the decline in the population of adjacent fillers mediated by one polymer layer $(\mathrm{BB}=1)$, and the growth in the population of adjacent fillers with no $(\mathrm{BB}=0)$ or two intervening polymer layers $(\mathrm{BB}=2)$ (Fig. 7). All these changes reduce the constraints on the polymer network. As seen in Fig. 11, the appearance of the yield drop also coincides with a marked disruption of the network of polymer molecules attached to filler particles at strains up to $\sim 20 \%$, followed by a gradual stabilisation of the network at higher strains.

This study has highlighted the crucial role of the dispersion of filler particles in the polymer matrix which directly affects the relative populations of the molecular structural motifs $(\mathrm{BB}=0,1,2)$ of filler particles, and which in turn determine the mechanical properties of the nanocomposite. Whilst it is widely accepted that higher surfaceto-volume ratio of the filler particles, for a given FVF, improves the mechanical properties of polymer nanocomposites, ${ }^{46-48}$ the influence of the shape of the filler particles on the structural motifs is less obvious, and it requires further research.

\section{Conflicts of interest}

There are no conflicts of interest to declare.

\section{Acknowledgements}

The authors acknowledge support from the Thomas Young Centre under grant TYC-101; NM was supported through a studentship in the Centre for Doctoral Training on Theory and Simulation of Materials at Imperial College London, funded by EPSRC under Grant No. EP/G036888/1 and by Baker Hughes GE. Thanks to David Curry, John Stevens, Jimmy Eason and Helmut Benning from Baker Hughes GE, to Imperial College High Performance Computing Service (DOI: 10.14469/hpc/2232), and to the UK's HEC Materials Chemistry Consortium, which is funded by EPSRC (EP/L000202), this work used the ARCHER UK National Supercomputing Service (http://www.archer.ac.uk).

\section{References}

1 F. Laoutid, L. Bonnaud, M. Alexandre, J. M. Lopez-Cuesta and P. Dubois, New prospects in flame retardant polymer materials: From fundamentals to nanocomposites, Mater. Sci. Eng., $R$, 2009, 63, 100-125, DOI: 10.1016/j.mser.2008.09.002.

2 D. Porter, E. Metcalfe and M. J. K. Thomas, Nanocomposite fire retardants - a review, Fire Mater., 2000, 24, 45-52, DOI: 10.1002/ (SICI)1099-1018(200001/02)24:1 < 45::AID-FAM719>3.0.CO;2-S.
3 J. M. Yeh, C. P. Chin and S. Chang, Enhanced corrosion protection coatings prepared from soluble electronically conductive polypyrrole-clay nanocomposite materials, J. Appl. Polym. Sci., 2003, 88, 3264-3272, DOI: 10.1002/app.12146.

4 J.-M. Yeh, C.-L. Chen, Y.-C. Chen, C.-Y. Ma, K.-R. Lee, Y. Wei and S. Li, Enhancement of corrosion protection effect of poly(o-ethoxyaniline) via the formation of poly(o-ethoxyaniline)-clay nanocomposite materials, Polymer, 2002, 43, 2729-2736, DOI: 10.1016/S0032-3861(02)00005-8.

5 S. Boutaleb, F. Zaïri, A. Mesbah, M. Naït-Abdelaziz, J. M. Gloaguen, T. Boukharouba and J. M. Lefebvre, Micromechanicsbased modelling of stiffness and yield stress for silica/polymer nanocomposites, Int. J. Solids Struct., 2009, 46, 1716-1726, DOI: 10.1016/j.jjsolstr.2008.12.011.

6 P. Podsiadlo, A. K. Kaushik, E. M. Arruda, A. M. Waas, B. S. Shim, J. Xu, H. Nandivada, B. G. Pumplin, J. Lahann, A. Ramamoorthy and N. A. Kotov, Ultrastrong and Stiff Layered Polymer Nanocomposites, Science, 2007, 318, 1-4, DOI: 10.1126/science.114317.

7 J. R. Potts, D. R. Dreyer, C. W. Bielawski and R. S. Ruoff, Graphene based polymer nanocomposites, Polymer, 2011, 52, 5-25, DOI: 10.1016/j.polymer.2010.11.042.

8 B. J. Ash, L. S. Schadler and R. W. Siegel, Glass transition behavior of alumina/polymethylmethacrylate nanocomposites, Mater. Lett., 2002, 55, 83-87, DOI: 10.1016/S0167577X(01)00626-7.

9 D. Fragiadakis, P. Pissis and L. Bokobza, Glass transition and molecular dynamics in poly(dimethylsiloxane)/silica nanocomposites, Polymer, 2005, 46, 6001-6008, DOI: 10.1016/j.polymer.2005.05.080.

10 P. Rittigstein, R. D. Priestley, L. J. Broadbelt and J. M. Torkelson, Model polymer nanocomposites provide an understanding of confinement effects in real nanocomposites, Nat. Mater., 2007, 6, 278-282, DOI: 10.1038/nmat1870.

11 B. Vorselaars, A. V. Lyulin and M. A. J. Michels, Deforming glassy polystyrene: Influence of pressure, thermal history, and deformation mode on yielding and hardening, J. Chem. Phys., 2009, 130, 074905, DOI: 10.1063/1.3077859.

12 L. Huang, X. Yang, X. Jia and D. Cao, Fracture mechanism of amorphous polymers at strain fields, Phys. Chem. Chem. Phys., 2014, 16, 24892-24898, DOI: 10.1039/c4cp03120b.

13 J. Li, T. Mulder, B. Vorselaars, A. V. Lyulin and M. A. J. Michels, Monte Carlo simulation of uniaxial tension of an amorphous polyethylene-like polymer glass, Macromolecules, 2006, 39, 7774-7782, DOI: 10.1021/ma061042w.

14 D. Hossain, M. Tschopp, D. Ward, J. Bouvard, P. Wang and M. Horstemeyer, Molecular dynamics simulations of deformation mechanisms of amorphous polyethylene, Polymer, 2010, 51, 6071-6083, DOI: 10.1016/j.polymer.2010.10.009.

15 S. Lee and G. C. Rutledge, Plastic deformation of semicrystalline polyethylene by molecular simulation, Macromolecules, 2011, 44, 3096-3108, DOI: 10.1021/ma1026115.

16 R. S. Hoy and M. O. Robbins, Strain hardening in polymer glasses: Limitations of network models, Phys. Rev. Lett., 2007, 99, 1-4, DOI: 10.1103/PhysRevLett.99.117801.

17 R. S. Hoy and M. O. Robbins, Strain hardening of polymer glasses: Entanglements, energetics, and plasticity, Phys. Rev. 
E: Stat., Nonlinear, Soft Matter Phys., 2008, 77, 1-14, DOI: 10.1103/PhysRevE.77.031801.

18 R. S. Hoy and M. O. Robbins, Strain hardening in bidisperse polymer glasses: Separating the roles of chain orientation and interchain entanglement, J. Chem. Phys., 2010, 131, 244901, DOI: 10.1063/1.3276800.

19 R. S. Hoy and C. S. O'hern, Viscoplasticity and large-scale chain relaxation in glassypolymeric strain hardening, Phys. Rev. E: Stat., Nonlinear, Soft Matter Phys., 2010, 82, 1-10, DOI: 10.1103/PhysRevE.82.041803.

20 K. Nayak, D. J. Read, T. C. B. McLeish, P. J. Hine and M. Tassieri, A coarse-grained molecular model of strain-hardening for polymers in the marginally glassy state, J. Polym. Sci., Part B: Polym. Phys., 2011, 49, 920-938, DOI: 10.1002/polb.22263.

21 M. Khawaja, A. Sutton and A. Mostofi, Molecular Simulation of Gas Solubility in Nitrile Butadiene Rubber, J. Phys. Chem. B, 2016, 121, 287-297.

22 G. Allegra, G. Raos and M. Vacatello, Theories and simulations of polymer-based nanocomposites: From chain statistics to reinforcement, Prog. Polym. Sci., 2008, 33, 683-731, DOI: 10.1016/j.progpolymsci.2008.02.003.

23 J. Liu, L. Zhang, D. Cao, J. Shen and Y. Gao, Computational Simulation of Elastomer Nanocomposites: Current Progress and Future Challenges, Rubber Chem. Technol., 2012, 85, 450-481, DOI: $10.5254 /$ rct.12.87966.

24 V. Ganesan and A. Jayaraman, Theory and simulation studies of effective interactions, phase behavior and morphology in polymer nanocomposites, Soft Matter, 2014, 10, 13-38, DOI: $10.1039 / \mathrm{c} 3 \mathrm{sm} 51864 \mathrm{~g}$.

25 A. Karatrantos, N. Clarke and M. Kröger, Modeling of Polymer Structure and Conformations in Polymer Nanocomposites from Atomistic to Mesoscale: A Review, Polym. Rev., 2016, 3724, 1-44, DOI: 10.1080/15583724.2015.1090450.

26 K. Kremer and G. S. Grest, Dynamics of entangled linear polymer melts: A moleculardynamics simulation, J. Chem. Phys., 1990, 92, 5057, DOI: 10.1063/1.458541.

27 J. T. Kalathi, S. K. Kumar, M. Rubinstein and G. S. Grest, Rouse mode analysis of chain relaxation in homopolymer melts, Macromolecules, 2014, 47, 6925-6931.

28 G. Raos, M. Moreno and S. Elli, Computational experiments on filled rubber viscoelasticity: What is the role of particleparticle interactions?, Macromolecules, 2006, 39, 6744-6751, DOI: $10.1021 / \mathrm{ma061008h}$.

29 G. Raos and M. Casalegno, Nonequilibrium simulations of filled polymer networks: Searching for the origins of reinforcement and nonlinearity, J. Chem. Phys., 2011, 134, 054902, DOI: 10.1063/1.3537971.

30 J. Shen, J. Liu, Y. Gao, X. Li and L. Zhang, Elucidating and tuning the strain-induced non-linear behavior of polymer nanocomposites: a detailed molecular dynamics simulation study, Soft Matter, 2014, 10, 5099-5113, DOI: 10.1039/c4sm00233d.

31 J. Shen, J. Liu, H. Li, Y. Gao, X. Li, Y. Wu and L. Zhang, Molecular dynamics simulations of the structural, mechanical and visco-elastic properties of polymer nanocomposites filled with grafted nanoparticles, Phys. Chem. Chem. Phys., 2015, 17, 7196-7207, DOI: 10.1039/c4cp05520a.
32 J. Liu, S. Wu, L. Zhang, W. Wang and D. Cao, Molecular dynamics simulation for insight into microscopic mechanism of polymer reinforcement, Phys. Chem. Chem. Phys., 2011, 13, 518-529, DOI: 10.1039/c0cp00297f.

33 G. S. Grest and K. Kremer, Molecular dynamics simulation for polymers in the presence of a heat bath, Phys. Rev. A: At., Mol., Opt. Phys., 1986, 33, 3628-3631, DOI: 10.1103/PhysRevA.33.3628.

34 J. Liu, D. Cao and L. Zhang, Molecular dynamics study on nanoparticle diffusion in polymer melts: a test of the StokesEinstein law, J. Phys. Chem. C, 2008, 112, 6653-6661.

35 J. S. Smith, D. Bedrov and G. D. Smith, A molecular dynamics simulation study of nanoparticle interactions in a model polymer-nanoparticle composite, Compos. Sci. Technol., 2003, 63, 1599-1605.

36 J. D. Weeks, D. Chandler and H. C. Andersen, Role of repulsive forces in determining the equilibrium structure of simple liquids, J. Chem. Phys., 1971, 54, 5237-5247.

37 S. Plimpton, Fast Parallel Algorithms for Short-Range Molecular Dynamics, 1995, http://www.sciencedirect.com/science/ article/pii/S002199918571039X.

38 J. Liu, S. Wu, D. Cao and L. Zhang, Effects of pressure on structure and dynamics of model elastomers: a molecular dynamics study, J. Chem. Phys., 2008, 129, 154905, DOI: 10.1063/1.2996009.

39 N. Molinari and S. Angioletti-Uberti, Nanoparticle Organization Controls Their Potency as Universal Glues for Gels, Nano Lett., 2018, 18, 3530-3537.

40 E. Kucukpinar and P. Doruker, Molecular simulations of gas transport in nitrile rubber and styrene butadiene rubber, Polymer, 2006, 47, 7835-7845, DOI: 10.1016/j.polymer.2006.08.062.

41 N. Molinari, M. Khawaja, A. P. Sutton and A. A. Mostofi, Molecular Model for HNBR With Tunable Cross-Link Density, J. Phys. Chem. B, 2016, 120, 12700-12707, DOI: 10.1021/ acs.jpcb.6b07841.

42 L. Yang, D. J. Srolovitz and A. F. Yee, Extended ensemble molecular dynamics method for constant strain rate uniaxial deformation of polymer systems, J. Chem. Phys., 1997, 107, 4396, DOI: 10.1063/1.474781.

$43 \mathrm{~J}$. Gao and J. H. Weiner, Simulated polymer melt stress relaxation. II. Search for entanglements, J. Chem. Phys., 1995, 103, 1621, DOI: 10.1063/1.469733.

44 J. Liu, J. Shen, Y. Gao, H. Zhou, Y. Wu and L. Zhang, Detailed simulation of the role of functionalized polymer chains on the structural, dynamic and mechanical properties of polymer nanocomposites, Soft Matter, 2014, 10, 8971-8984, DOI: 10.1039/C4SM02005G.

45 J. B. Hooper and K. S. Schweizer, Contact aggregation, bridging, and steric stabilization in dense polymer-particle mixtures, Macromolecules, 2005, 38, 8858-8869.

46 A. Adnan, C. Sun and H. Mahfuz, A molecular dynamics simulation study to investigate the effect of filler size on elastic properties of polymer nanocomposites, Compos. Sci. Technol., 2007, 67, 348-356.

47 A. J. Crosby and J.-Y. Lee, Polymer nanocomposites: the "Nano" effect on mechanical properties, Polym. Rev., 2007, 47, 217-229.

$48 \mathrm{H}$. Alter, Filler particle size and mechanical properties of polymers, J. Appl. Polym. Sci., 1965, 9, 1525-1531. 